\title{
Research on garment pattern design based on fractal graphics
}

Weijie Wang ${ }^{1}$, Gaopeng Zhang ${ }^{1 *}$, Luming Yang ${ }^{2}$ and Wei Wang ${ }^{2}$

\begin{abstract}
This paper firstly analyzes the basic principle of generating fractal art graphics and the artistic features of graphics and then uses scientific visualization method to innovate and improve the theoretical model used in this paper. The generation principle and graphic characteristics of fractal graphics of complex dynamic system and L-system are mainly analyzed, and two kinds of art graphics - flower art graphics and geometric art graphics - have been developed. On this basis, the generated artistic figures are designed for the second time and then applied to the design of clothing patterns. By using MATLAB programming software to generate art graphics conforming to a specific style, combined with image processing software Photoshop to process and redesign the generated graphics, these art graphics can assist the design of clothing printing patterns and make patterns applicable for clothing fabrics. Finally, the fractal pattern theory is applied to silk scarves design and clothing fabric design through digital printing technology, which can fully reflect the practicability and superiority of clothing pattern design based on the fractal theory. Based on the experimental result, it shows that it is completely feasible to design clothing fabric printing patterns based on fractal theory, and the unusual artistic patterns designed have very considerable practical value. In addition, this method encourages creativity in the garment pattern design process and accelerates new design generation.
\end{abstract}

Keywords: Clothing design, Computer graphics, Fractal theory, Complex dynamical system, L-system, Paper-cut pattern

\section{Introduction}

With the rapid development of information technology, fashion information is spreading faster and faster. Countless fashion brands and popular information are instilled in people's minds, affecting their dressing patterns [1]. In modern society, clothing is not only a tool for people to keep warm from the cold, but also an indispensable ornament in pursuit of individuality [2]. Therefore, clothing design plays an important role in guiding consumption and beautifying life, of which pattern design is a crucial link.

As we all know, art and mathematics have a profound connection. Artists can use mathematical ideas to create more profound art, and esthetic factors play a particularly important role in the study of mathematics; so, mathematics is regarded as an art usually [3]. The development of modern computer technology is to combine art and mathematics closely, and the beauty contained in mathematical formulas and theorems is gradually discovered and

\footnotetext{
* Correspondence: zgpscu1967@163.com

${ }^{1}$ Faculty of Textile and Garment Engineering, College of Light Industry,

Textile and Food Engineering, Sichuan University, Chengdu 610065, China

Full list of author information is available at the end of the article
}

utilized by people in the field of art. Therefore, garment pattern designers began to focus on science and technology and mathematics, hoping that they could provide new design resources for garment pattern design [4]. The emergence of mathematical art graphics has injected fresh vitality into traditional clothing patterns.

In all kinds of mathematical forms, Euclid's geometry shows a strict esthetic order from strict symmetry to the use of golden ratio, and this order can be found everywhere in people's daily life, such as building design and city planning. However, with the deepening of scientific exploration and esthetic changes, the beauty of this form, which has lasted for thousands of years, can no longer meet people's esthetic needs. After all, European geometry can only accurately depict simple patterns rather than patterns in nature. After the middle of the nineteenth century, scholars began to explore various kinds of irregular phenomena in nature and discovered that many things are not regular, such as the outline of clouds, coastline, shape of lightning, and these irregular figures are obviously pale and dynamical less to describe with traditional Euclidean geometry [5]. Under this environment, the 
fractal geometry founded by the famous American French mathematician Benoit Mandelbrot came into being [6]. Fractal geometry is a subject that takes irregular geometry as its research object, in which nature is regarded as a fine structure with infinite nesting levels and maintaining some self-similar properties at different scales. Therefore, fractal geometry is also called geometry describing nature [7]. After that, fractal design, fractal information, fractal art, and other applications have been derived from fractal geometry, thus forming a very popular and active new theory-fractal theory [8].

The traditional pattern design and production are all done by hand. First, designers construct an artistic representation in the brain and then use different materials and techniques to display the artistic representation concretely [9]. However, due to the limitations of the designer's creative ability and imagination, the created pattern will also have certain deficiencies in form and type, and the creation cycle is long and the subsequent modification process is very cumbersome. Besides, there are still many pattern designs that are difficult or impossible to draw by hand. Traditional pattern design and production methods no longer meet the life rhythm and market demands of modern society. In order to enrich the form of clothing patterns and meet people's changeable psychological needs, designers need to find new ways suitable for clothing pattern design. The fractal pattern is drawn by computer graphics technology, which is a self-similar pattern with infinite fine structure and an embodiment of unpredictable beauty [10], and the use of fractal can create a variety of artistic patterns with high resolution. Fractal graphics can be generated by computer programming; by changing parameters, various artistic patterns with different shapes can be generated. As a new design resource, fractal has attracted people's attention in the field of design.

Based on the aforementioned research background, this paper firstly analyzes the fractal theory and L-system generation method in Section 2. Based on the fractal theory described in Section 2, the computer pattern technology is combined with complex dynamical system theory to generate fractal art graphics by computer programming in Section 3, and the generation methods of clothing pattern in complex dynamical system are analyzed in detail. Combining with computer graphics technology, a series of source codes to generate geometric patterns that can meet people's esthetic needs are compiled in Section 4, to obtain the L-system design results of garment geometric patterns. Based on the aforementioned study results, a series of clothing pattern formation are presented in Section 5. Finally, the fractal pattern generation theory studied in this paper is applied to conduct the actual design of silk scarves and garment fabric patterns in Section 6. Section 7 concludes the paper.

\section{Method-fractal theory and L-system generation}

In 1975, the founder of fractal theory Mandelbrot created the word "fractal" based on the word of "frangere" in the Latin language, which has two meanings of irregular and broken [11]. However, there is no strict mathematical definition of fractal at present, and there is only descriptive definition. For example, the British mathematician Falconer pointed out in the book "Mathematical Basis and Application of Fractal Geometry" that fractal should be defined as a set F with the following properties:

$\mathrm{F}$ is irregular and cannot be traced with traditional geometric language; the fractal dimension defined by $\mathrm{F}$ in some way is usually larger than the topological dimension of F; F has a fine structure and contains the whole in any small scale; F usually has some self-similarity and may be similar in statistical sense; the definition of $F$ is usually very simple, perhaps recursive.

Fractal can be generally divided into two categories: random fractal and deterministic fractal, and the latter means that the same graph is still generated by repeating the same algorithm many times. Even if some random factors are added in the process of generating the graph, the obtained graph will remain unchanged. However, random fractal means that under the condition that the rules for generating fractal graphics are unchanged; the final generated graphics will be different due to the influence of random factors. Although random fractal has its own rules, the final generated graphics are unpredictable due to the introduction of random factors in the generation process, that is, the graphics produced by two operations in different time can have the same fractal dimension, but the shapes may be different, so random fractal has no repeatability.

There are mainly three methods to generate fractal graphics: complex iterative generation method, L-system generation method, and IFS generation method. At present, the L-system generation algorithm is commonly used for fractal pattern generation based on complex dynamic system, which is also the method used in this paper. The L-system was firstly used as a method to describe plant morphology and growth process and then developed into a method to simulate natural scenery in computer graphics, which was proposed by Danish biologist Aristid Lindenmayer in 1968 [12]. The working principle of L-system is very simple, which only operates on a few simple characters, of which core idea is string replacement. As a result, the L-system is actually a string rewriting system, based on which various fractal patterns are generated by generating a series of character strings, including not only many classical fractal patterns, but also simulate the shape of plants in nature, especially the branch structure of plants.

There are also several kinds of L-systems in detail. Generally, the so-called L-systems refer to defined 
L-systems, that is, DOL-system, which is the simplest type of graphical simulation system in the L-system family. The DOL-system is defined (D) and have zero interaction with the context (O) [13], which is an ordered set of three elements $\{v, p, w\} ; v$ represents alphabet, $v^{*}$ represents all non-empty word sets on $v, p$ belongs to $v \times v^{*}$ that is a finite set of production forms, and $w \in v^{*}$ is a non-empty word called axiom. A production form $(a, s) \in p$ can be written as $a \rightarrow s$, and $a$ and $s$ are precursors and successors of production forms respectively.

The string of the L-system is also called turtle graph, of which basic idea is presented as follows: a state of turtle is defined as $(x, y, \alpha)$, where $(x, y)$ representsthe position of turtle; the angle $\alpha$ is called the direction which is considered the direction the turtle faces. Given the angle increment $\delta$ and step $d$ of the turtle's twisting direction, the commands that the turtle can respond to are described by the following symbols:

(1) $f$ : move forward one step and draw a line, and the length is step d. After passing this command, the state of the turtle will change from $(x, y, \alpha)$ to $\left(x^{\prime}, y^{\prime}, \alpha\right)$, where $x=x+d \cdot \cos \alpha, y=y+d \cdot \sin \alpha$

(2) $h$ : move forward one step, and the length is step d. The state change is the same as that of $\mathrm{f}$ command, but the turtle does not leave any trace of crawling, that is, not draw a line

(3) $R$ : draw a line segment to the right according to the given step $d$

(4) $L$ : draw a line segment to the left according to a given step $d$

(5) [: put the current drawing format in the stack

(6)]: exit the stack and return to the drawing state before the left square bracket [

(7) +: rotate the angle $\delta$ to the left or counterclockwise direction. By this command, the turtle's next state will change from $(x, y, \alpha)$ to $(x, y, \alpha+\delta)$. The positive direction of the specified angle is counterclockwise and the negative direction is clockwise.

(8) -: rotate the angle to the right or clockwise, and by this command, the turtle's next state will change from $(x, y, \alpha)$ to $(x, y, \alpha-\delta)$.

If the transformation rule is applied to an initial string (or letter) many times, a longer string will be generated, this string will be used to draw a graph once called level 1, and $n$ times correspond to level $n$. Generally, the selected number of stages should not be too high, and level $2-8$ is usually selected. Once the following parameters are input in the process of drawing, the ever-changing fractal graph can be drawn: axiom (initial element), production formula (generator element), compression factor, iteration number, and angle increment $\delta$. To illustrate the generation process of the fractal graph, two examples are presented as follows:

Example 1: Given the initial angle is $0^{\circ}$, angle increment $\delta=90^{\circ}$, axiom $w: \mathrm{f}+\mathrm{f}+\mathrm{f}+\mathrm{f}$, generating formula $p: \mathrm{f} \rightarrow \mathrm{ff}-\mathrm{f}-\mathrm{f}-\mathrm{f}-\mathrm{f}+\mathrm{f}$, compression factor is $1 / 3$. The initial element is presented as Fig. 1(a) shows, and Fig. 1(b)-(d) are generated when the number of iterations $n=1,2$, and 3 respectively.

Example 2: The simple forward and backward format of the branch structure-fractal tree. Given the angle increment $\sigma=25.7^{\circ}$, axiom $w$ : f, generation formula $p 1$ : $\mathrm{f} \rightarrow \mathrm{h}[-\mathrm{f}][+\mathrm{f}] \mathrm{hf}, p 2: \mathrm{h} \rightarrow \mathrm{hh}$, compression factor $1 / 3$, and Fig. 2(a), (b), and (c) are generated respectively in the case of iteration times $n=1,2$, and 6 respectively.

\section{Design of clothing pattern in complex dynamical system}

Based on the fractal theory described in Section 2, the computer pattern technology is combined with complex dynamical system theory to generate fractal art graphics by computer programming in this section, and the generation of complex dynamical system and the methods of changing the color parts of graphics are analyzed in detail.

\subsection{Design method of garment printing pattern for complex dynamical system}

Most of the graphs generated based on Julia's set theory [14] have irregular shapes, fine structures, local and global similarities, symmetry across different scales, and so on. The flow chart of Julia set's pattern design is shown in Fig. 3, where $c$ is the given parameter, $Z_{0}$ is the initial point, $N$ is the maximum number of iterations, $R$ is the escape radius, and there are a total of $n \times m$ initial points. $x_{\min }, y_{\min }, x_{\max }$, and $y_{\max }$ are the minimum and maximum values on the $X$ - and $Y$ - axes respectively, and the iterative function can be changed accordingly. In Fig. 3, the initial point of iteration is given: $j=0(j \in(0, N)), x_{0}=x_{\min }+v_{x}$ $\cdot \Delta x, y_{0}=y_{\min }+v_{y} \cdot \Delta y$.

\subsection{Pattern design of complex dynamical system based on Julia set}

Flowers are most widely used in fashion design, of which shape, color, and posture are regarded by people as a symbol of good happiness [15], and therefore, flower graphics are widely used in clothing, home textiles, and other textiles as decorative patterns. This is not only because people love the beauty of flowers, but also in the design of textile patterns, flower patterns have the characteristics of strong flexibility and wide applicability. From the aspect of pattern composition, the flower image can be naturally and flexibly organized whether it is a single pattern or a combination pattern and a continuous strip or a planar extension.

Fractal art graphics are protean, which have different shapes and are a simulation of nature. In this section, the complex dynamical system is mainly used to carry out pattern design, and two types of common flower pattern based on Julia set are presented. 


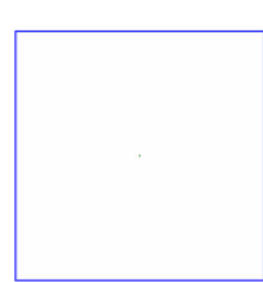

a) Initial Element

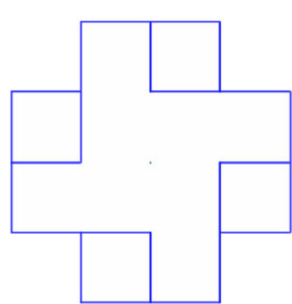

b) $n=1$

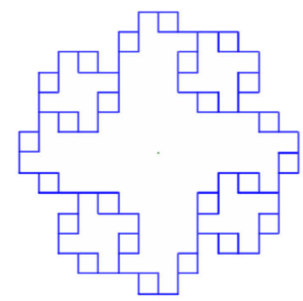

c) $n=2$

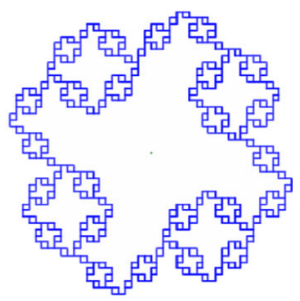

d) $n=3$

Fig. 1 L-system geometric graph

Triangle flower type. Based on Julia's plot program, a series of triangular patterns can be designed, of which pattern color is mainly warm as shown in Fig. 4. The three patterns in Fig. 4 are all generated by Julia's set, and their iterative functions are $Z=Z^{3}+\tan (c)+c, Z=Z^{3}+c, Z=Z^{3}+c$ respectively, and the value of parameter $c$ is given below the pattern.

Quadruple pattern. Figure 5 shows a series of four-corner patterns designed by using Julia's plot program, of which the first three patterns are pattern designed mainly with warm colors, and their iterative functions are: $(a) Z=Z^{4}+c, \quad Z=Z^{6}+c ; \quad(b) Z=Z^{4}+c, \quad Z=Z^{6}+c$; (c) $Z=Z^{6}+c, \quad Z=Z^{2}-0.5+0.2 i, \quad Z=Z^{4}+c$; the last three patterns are patterns designed with cold colors, of which functions are $Z=Z^{4}+c$. The corresponding parameter $c$ is marked under the pattern.

\section{L-system design of garment geometric patterns}

Based on the above fractal theory analysis, it can be seen that the graph generated by the L-system is linear. The graph generated by the L-system can not only simulate the growth of plants such as flowers, plants, and trees in nature, but also can generate various geometric figures with fine structures. Combining with computer graphics technology, a series of source codes to generate geometric patterns that can meet people's esthetic needs are compiled in this study.

\subsection{L-system pattern design method}

As mentioned above, once the influence factors of L-system plotting are determined, the fractal graph can be generated through repeated iterations of computer programs. The program flow chart of the L-system pattern is shown in Fig. 6.

\subsection{L-system pattern geometrical design}

Geometrical design and flower design are common themes in clothing design, of which rich and colorful decorative form is deeply loved by people [16], and it is an indispensable decorative form for beautifying life. The organizational foundation of geometrical design is composed of rehearsals and interweaves of points, lines, and faces, extending from a certain angle, distance, and direction, and organized into esthetic, visual, rigorous, and implicit forms of expression according to certain principles [17]. The geometrical design is the organic combination of various visual elements making people feel rich and unified, diverse, and organized, which is full of symbolism and form and exudes infinite artistic charm.

The loop iteration of L-system can be used to draw various geometric figures, which have different degrees of self-similarity, infinite mosaic, and fine structure, which cannot be compared with the traditional geometric figures [18]. As the oldest participant in clothing design elements, the concrete language has been

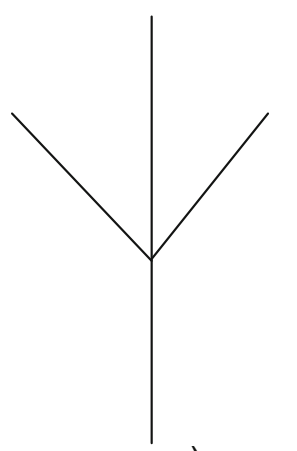

a) $n=1$

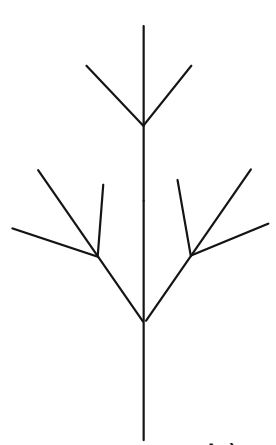

b) $n=2$

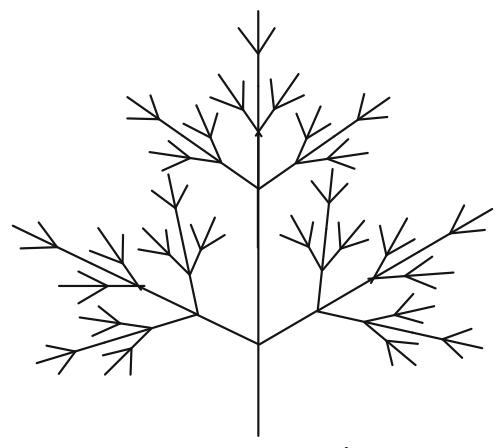

c) $n=6$

Fig. 2 L-system fractal tree 


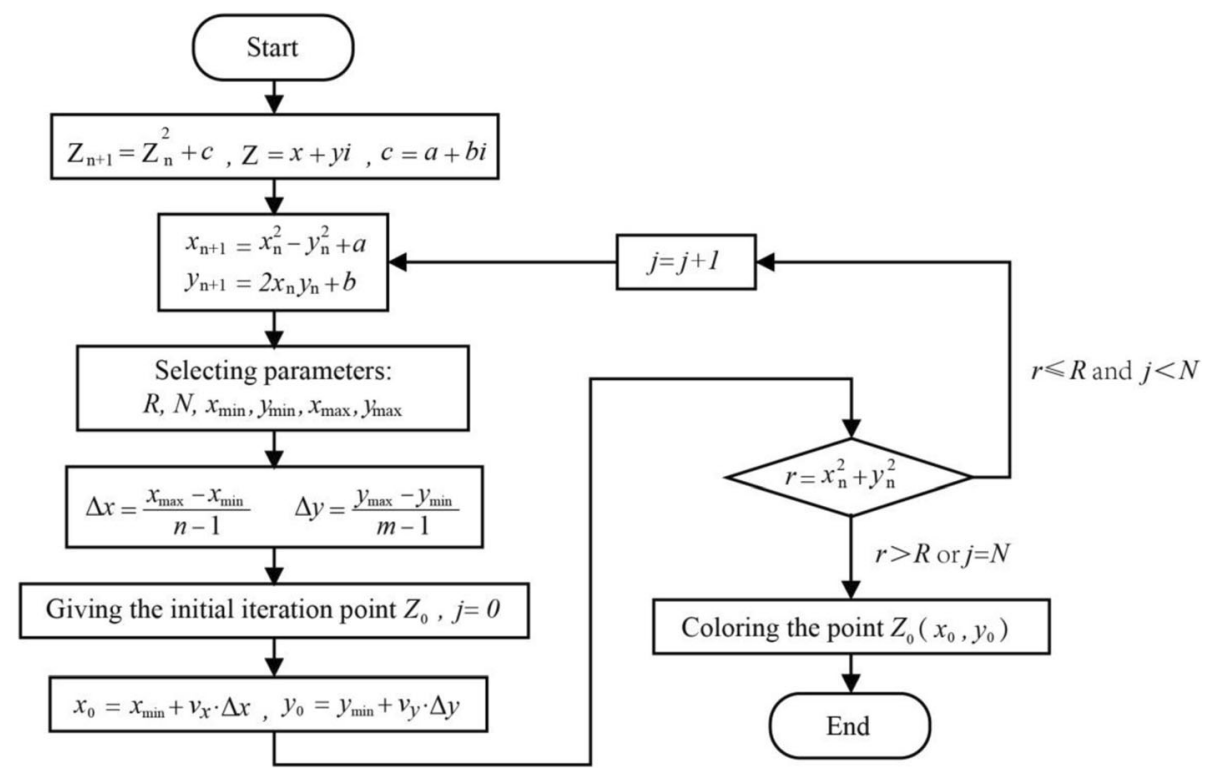

Fig. 3 Pattern design flow chart based on Julia set

put aside and a more simple way is used to reflect the pure form of modeling vocabulary and decorative forms in geometric patterns. According to the composition principle of the L-system, a program for generating geometric patterns is written using MATLAB language, and the following geometric patterns are designed [19].

Paper-cut patterns 1. All graphs in the graph have a common generation rule P: F+F--F-F--F--FF, the initial angle $\theta=0^{\circ}$, the angle increment $\delta=60^{\circ}$, and the step size is $1 / 3 \mathrm{~d}$, but the initial element and iteration number are different (Fig. 7). The initial elements of the four graphs (a), (b), (c), and (d) are $w 1: \mathrm{F}++\mathrm{F}++\mathrm{F}$, which is a regular triangle with the initial element twist angle $\alpha=60^{\circ}$, and the iteration times are $n=1$, $n=2, n=3$, and $n=4$, respectively; (e), (f), (g), and (h) the initial elements of the four graphs are $w 2: \mathrm{F}+\mathrm{F}+\mathrm{F}+\mathrm{F}$, which is a square, $\alpha=90^{\circ}$, and the iteration times are $n=1, n=2, n=3$, and $n=4$ respectively.
Paper-cut patterns 2. In Fig. 8, all graphics have a common generation rule P: F + F--FF--F--FF--F + F, the initial angle $\theta=0^{\circ}$, angle increment $\delta=60^{\circ}$, and step size is $1 / 4 \mathrm{~d}$, but the initial element and iteration number are different. The initial elements of the graphs (a), (b), (c), and (d) are w1: $\mathrm{F}++\mathrm{F}++\mathrm{F}$, and the initial element twist angle $\alpha=60^{\circ}$. The iteration times are $n=1, n=2, n=3$, and $n=4$ respectively; (e), (f), (g), and (h) the initial elements of the four graphs are $w 2: \mathrm{F}+\mathrm{F}+\mathrm{F}+\mathrm{F}, \alpha=90^{\circ}$, and the iteration times are $n=1, n=2, n=3$, and $n=4$ respectively.

\section{The formation of clothing patterns 5.1 Individual pattern}

The most basic pattern unit and organization form in pattern design is the unit pattern, which mainly refers to the pattern that can exist and be used independently in shape and structure without connection and continuity with other forms [20]. There is no restriction on the appearance of

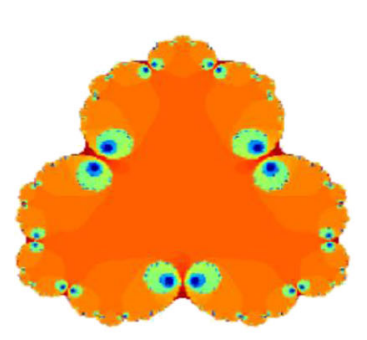

a) $c=-0.196$

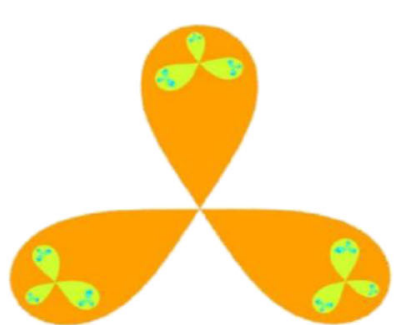

b) $c=-2+0.49 i$

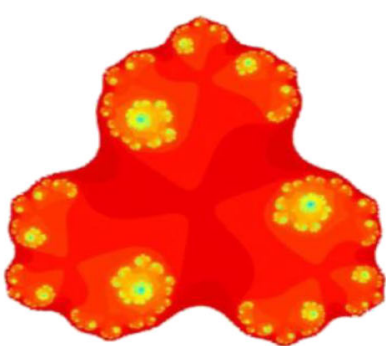

c) $c=0.649+0.175 i$

Fig. 4 Triangle pattern 


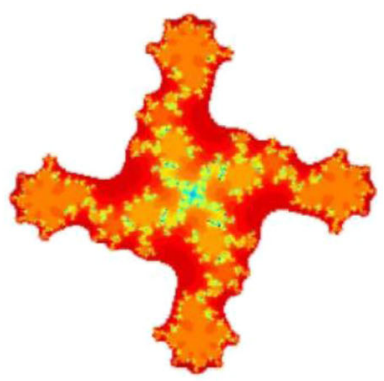

a) $c=-1+0.134 i$

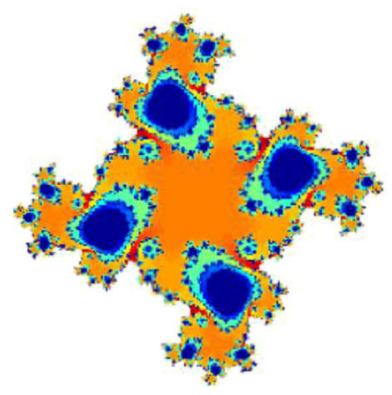

d) $c=-0.643+0.353 i$

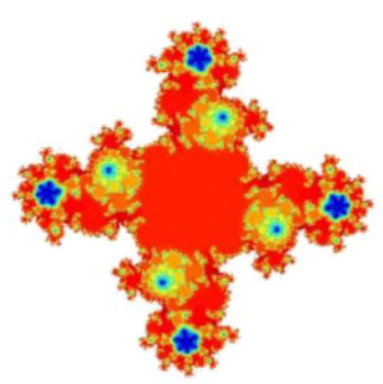

b) $c=-0.8+0.143 i$

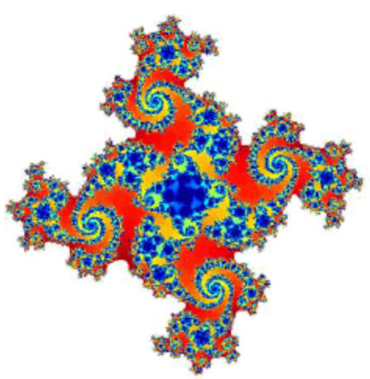

e) $c=-0.663-0.352 i$

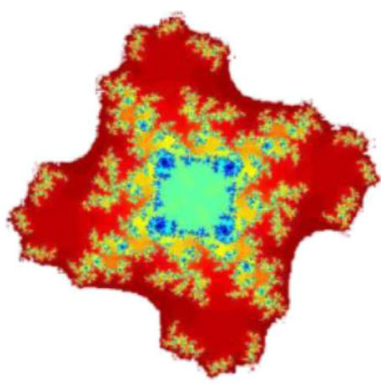

C) $c=-0.5+0.49 i$

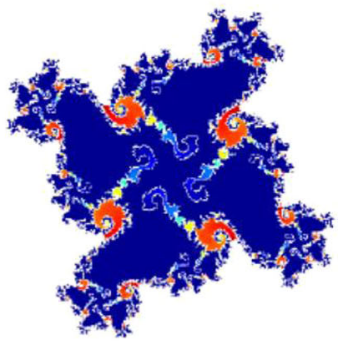

f) $c=0.333-0.172 i$

Fig. 5 Quadruple pattern

individual patterns, which can be combined with other patterns to form suitable patterns or continuous patterns in pattern design and can also be used as decorative patterns alone. The structure of the individual patterns should be clear and can directly reflect the movement of the pattern shape, which is the concrete embodiment of the image spirit and interest. The composition of the individual patterns should be clear in primary and secondary [21], strengthening the main part, and the secondary part often plays the role of filling and foil. Individual patterns are the basis of all patterns, which can be divided into two forms: homogeneous and balanced in form and layout.

Homogeneous type is also called symmetrical type, which is characterized by regular pattern structure, including absolute symmetry and relative symmetry [22]. Absolute symmetry is based on the central axis or center point, and patterns are symmetrical up and down, left and right, or in multiple faces around the central axis or center point, so that patterns form an absolute alignment structure. According to the different angles of symmetry, it can be divided into two forms: left-right symmetry and rotational symmetry as shown in Fig. 9.

The characteristic of balance type is not limited by the center point and the center axis in modeling and spatial arrangement, and its structural form is more flexible and free, but the size and color of the internal elements remain balanced in the whole; this kind of graphic has a prominent theme, relaxed image, and flexible style.

\subsection{Suitable pattern}

Suitable pattern refers to the pattern composition suitable for a specific shape in the shape, among which the common shapes are triangle, circle, square, hexagon, semicircle, plum blossom, peach, gourd, etc. The suitable patterns are equipped with beautiful forms, vivid images, rigorous structure, and rich colors, which can be arranged and combined as basic patterns or used alone [23].

The structure for suitable patterns can also be divided into two types: homogeneous and balanced types. Homogeneous type includes vertical type, centripetal type, centrifugal type, centripetal centrifugal type, rotary type, and conversion type. The balanced form of composition is relatively free, but attention should be paid to the relationship between spatial layout forms such as density, reality and direction, and the image before each other. As shown in Fig. 10, the suitable pattern can be classified as complex dynamical system pattern and L-system geometric pattern.

\subsection{Continuous pattern}

The continuous pattern develops in two or four directions with a simple pattern unit, becoming a strip-shaped pattern or a pattern surface with a certain length. The biggest characteristic of continuous patterns is the continuity of patterns, 


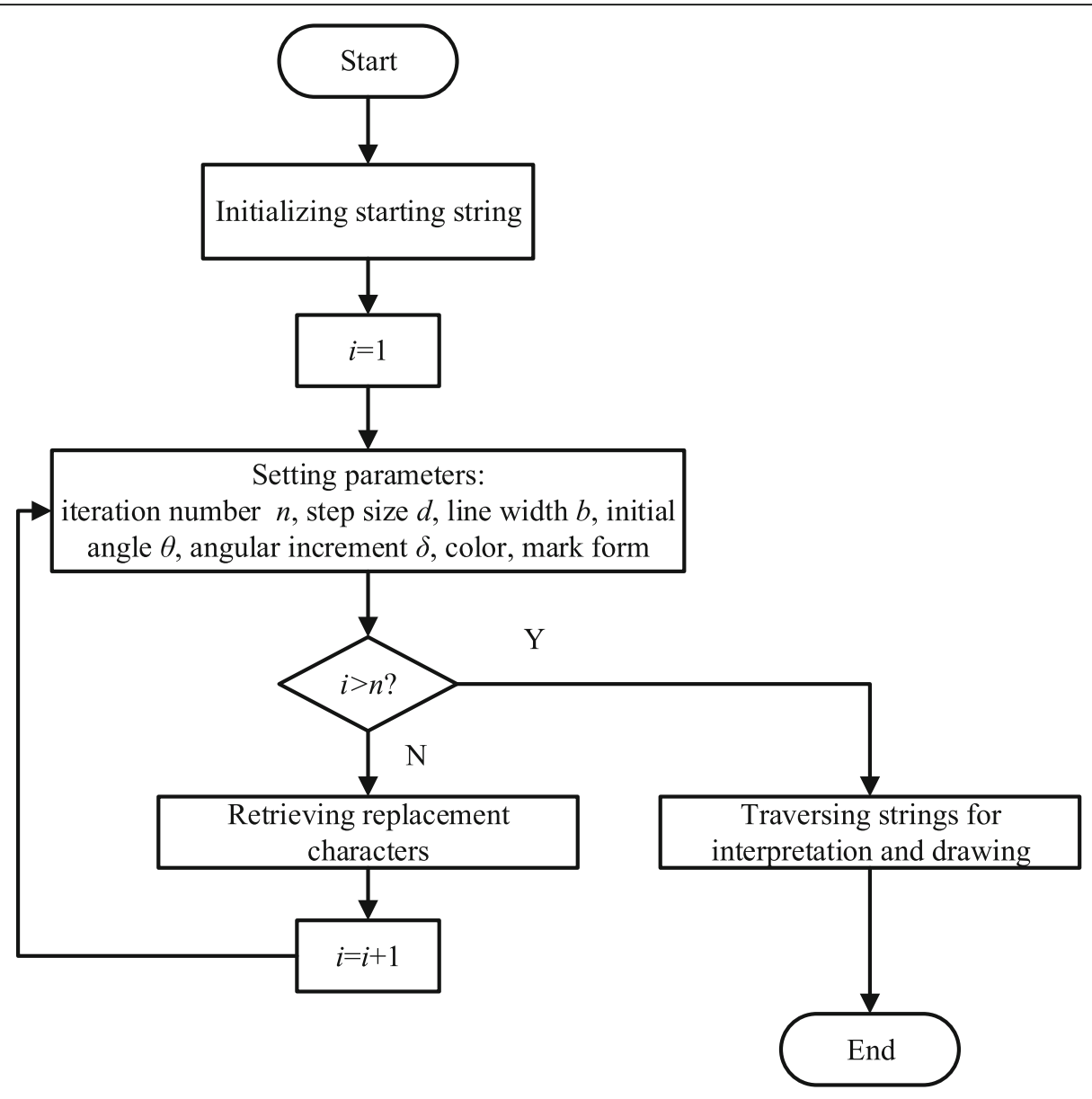

Fig. 6 The program flow chart of L-system pattern

with rhythm and rhythm beauty. Continuous patterns have a wide range of applications, such as architecture, decoration, textile, clothing, ceramics, and other design fields. The continuous pattern of the pattern is mainly two-dimension series pattern [24].

The two-dimension series pattern is also called ribbon patterns or lace, which is a strip-shaped pattern with unit patterns arranged up and down or left and right continuously and repeatedly according to a certain rule. The skeleton of the two-dimension series is designed according to the principle of repetitive rhythm, reflecting a strong sense of rhythm and rule. In the process of designing, the importance should be attached to the relationship between the structural continuity and units and density and movement of patterns, to make the forms naturally interpenetrate with each other, and avoid stiff connection and lacking coherence and integrity. There are three forms in the two-dimension series pattern, which are scatter type, vertical type, and corrugated type [25].

The scatter type usually appears repeatedly on the screen according to a certain direction and distance with one or several basic patterns as elements, of which arrangement features are point-shaped. In scatter-type two-dimension series pattern design, the relationship between points should be handled carefully: points cannot be too far away from each other, or else, there is no sense of echo; meanwhile, the distance should not be too short, or else, the picture will be too compact, that can result in stiff visual feeling. Therefore, the designed density should be reasonable as shown in Fig. 11.

The individual patterns are arranged and continued in a vertical manner, of which organization is relatively simple, and only one or more than two kinds of individual patterns are generally arranged in sequence. The composition of vertical patterns is characterized by a stable center of gravity with the meaning of solemn and loyal the two-dimension series vertical type pattern is presented as shown in Fig. 12.

In the corrugated-type pattern design, a unit element pattern is usually taken as the parent, which repeatedly appears on the screen according to a certain direction and distance, forming a wavy organizational form of the curve. The corrugated type includes straight corrugation and curved corrugation, and the former is the 

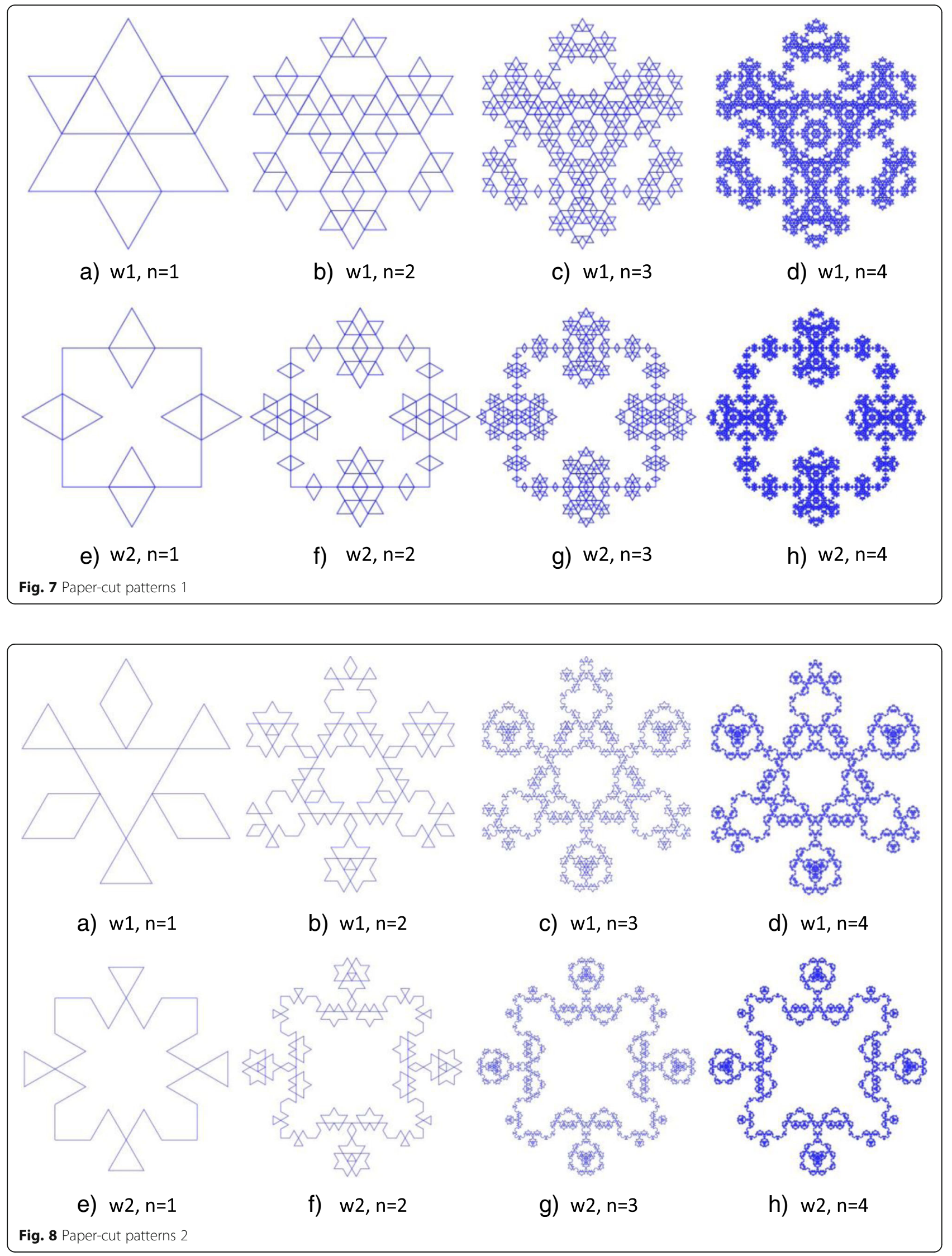


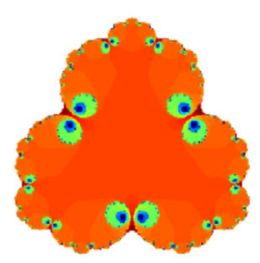

a) bilateral symmetry
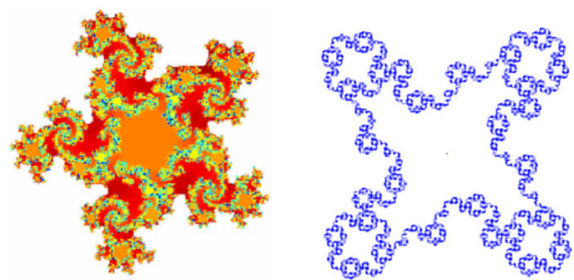

b) Rotational symmetry

Fig. 9 Symmetric individual patterns

connection of unit patterns with straight lines, in which straight turning part will form obvious corners and the whole pattern will be strong. Curved corrugation unit patterns are connected by undulating waves with smooth, free and soft forms, which are shown in Fig. 13.

\section{Results and discussions-design and application of clothing printing pattern based on computer graphics pair}

Researchers have made a lot of achievements in the research of using fractal theory and computer technology to directly generate fractal decorative patterns. However, less research has been done on the further composition design of these patterns. The composition of clothing patterns is to deform, rotate, and copy the basic elements, then arrange them according to certain rules and combine them into different composition forms. The basic pattern forms the structural feature of repeated and continuous clothing patterns through repeated circulation and connection and extension. Therefore, in view of the shortcomings of the current fractal art pattern directly generated in the form of clothing pattern, this section is focused on the design and application of complex dynamic system pattern and L-system geometry pattern in the form of clothing pattern.

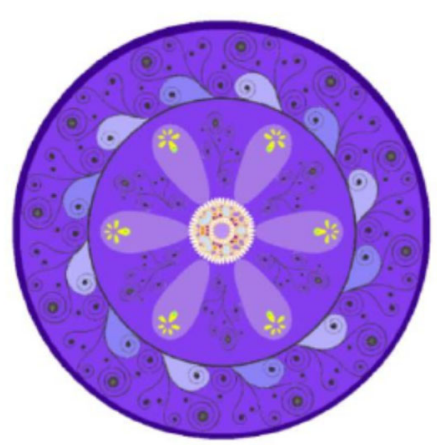

a) Complex Dynamical System Pattern - Circle

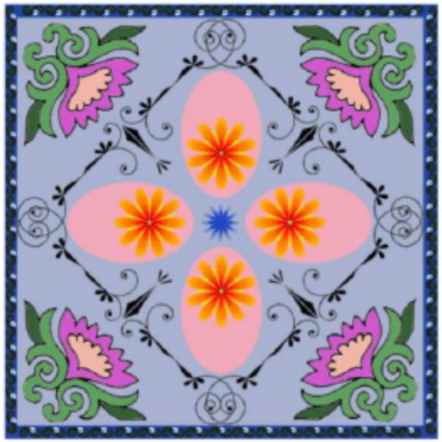

c) Complex Dynamical System Pattern - Square

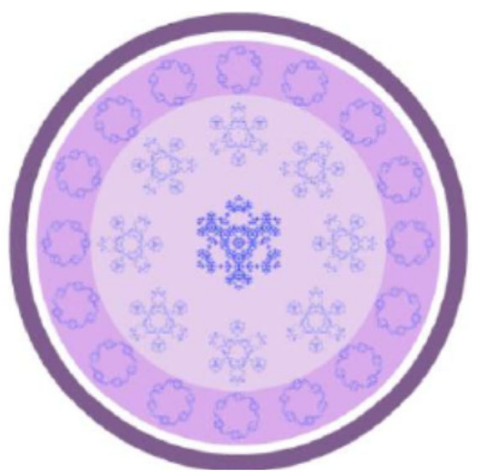

b) L-System Geometry - Circle

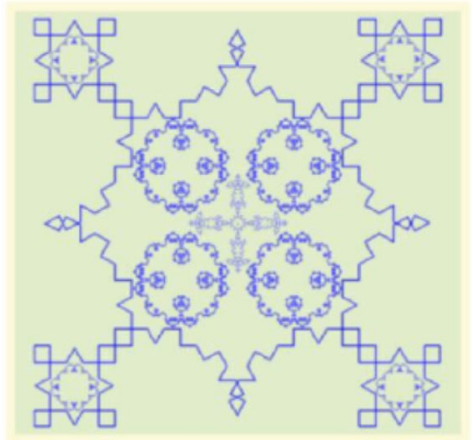

d) L-System Geometry - Square

Fig. 10 Suitable pattern 


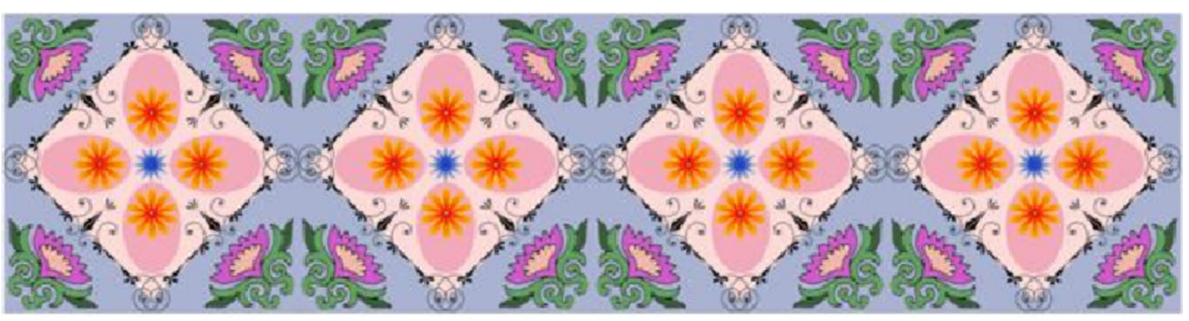

a) The Two-Dimension Series Scatter Type Pattern of Complex Dynamical System

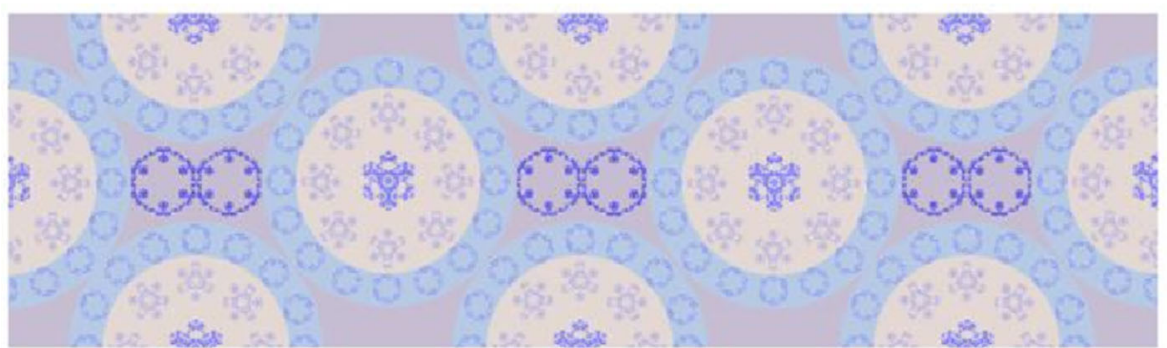

b) The Two-Dimension Series Scatter Type Pattern of L-System

Fig. 11 The two-dimension series scatter-type pattern

\subsection{Application of fractal pattern in silk scarves printing pattern}

Silk scarves are extremely important clothing accessories in people's daily life, which are equipped with unique cultural characteristics and influenced by esthetic tastes and customs. Pattern is the soul of silk scarves and also the crucial factor for consumers to choose silk scarves. The traditional silk scarves mainly include exquisite printing patterns, various plants and geometry. In this study, the fractal art pattern with a more contemporary sense is applied to the design of silk scarves, bringing new elements to the design of silk scarves.

The commonly used square towels are $110 \mathrm{~cm} \times 110 \mathrm{~cm}$, $88 \mathrm{~cm} \times 88 \mathrm{~cm}$, and $52 \mathrm{~cm} \times 52 \mathrm{~cm}$, and long towels are $180 \mathrm{~cm} \times 52 \mathrm{~cm}, \quad 160 \mathrm{~cm} \times 35 \mathrm{~cm}$, and $140 \mathrm{~cm} \times 26 \mathrm{~cm}$. According to the form of clothing pattern and the related factors of pattern design, the fractal pattern is used to design silk scarves in this study.
Symmetry is a common pattern form, and the figure with symmetrical structure makes people feel relatively neat and stable. Once the two sides of the symmetry axis are basically consistent visually and a balanced state can be reached, it can show a lively, varied, and dynamic artistic feeling. Based on the basic patterns (Fig. 14a and b) and other auxiliary patterns, the silk scarf pattern is designed as shown in Fig. 14c according to the symmetry beauty principle of pattern design.

A square silk scarf with a size of $88 \mathrm{~cm} \times 88 \mathrm{~cm}$ is designed by using the basic pattern diagram of the complex dynamical system. The individual patterns designed by the computer are shown in Fig. 15(a), in which the color of orange is used as the main color and complementary blue as appropriate to reconcile the monotony of the picture, which makes the patterns bright and vigorous but not too exciting, sharp, and dazzling. According to the formal beauty rules of pattern design, the individual patterns are

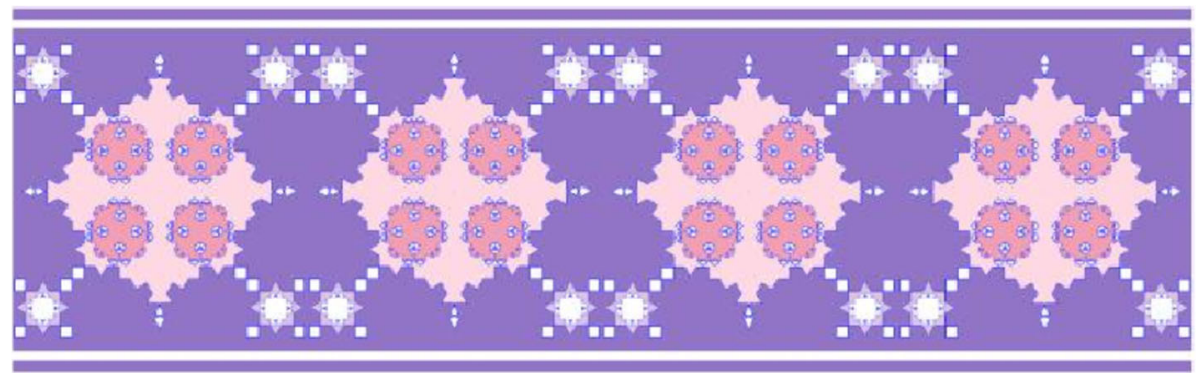

Fig. 12 The two-dimension series vertical-type pattern 


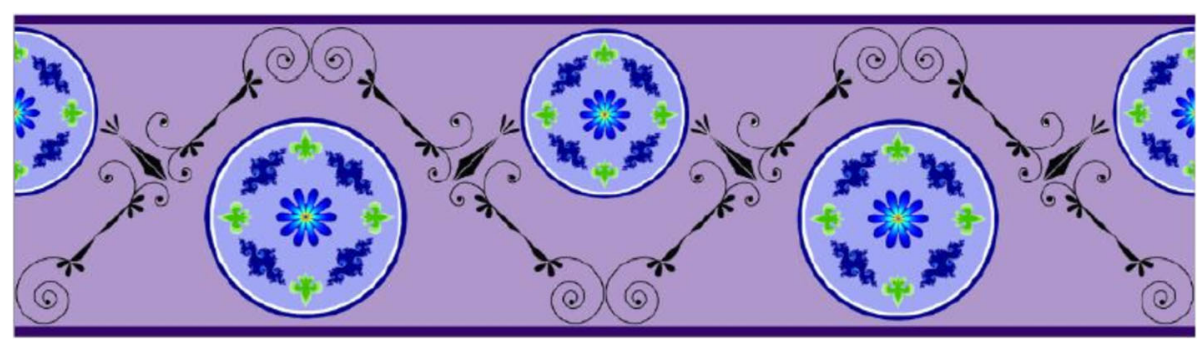

Fig. 13 The two-dimension series corrugated-type pattern

arranged in scattered square patterns to obtain the square silk scarf pattern as shown in Fig. 15(b). The contrast color configuration can produce a strong and lively, bright, and eye-catching feeling. Finally, the silk scarf pattern is made into a real object through digital printing, and the real object diagram as well as the wearing effect diagram are obtained, which are presented in Fig. 15(c) and (d) respectively.

\subsection{Application of fractal pattern in garment fabric printing pattern}

Patterns are widely used in the field of clothing, especially in the design of clothing fabrics. Patterns, materials, colors, and styles together constitute the four elements of clothing fabric design. Nowadays, there is a decreasing trend in garment fabrics without patterns on the market, because patterns can enhance fashion sense and artistic sense of clothing and also become a special requirement for people to pursue the perfection of clothing, which will be integrated into the design of fashion fabrics more and more and have a wide application prospect.

From the point of view of clothing, the composition of clothing patterns is mostly based on the continuous pattern of four sides, and various patterns can be produced through the orderly repetition, transformation or cross

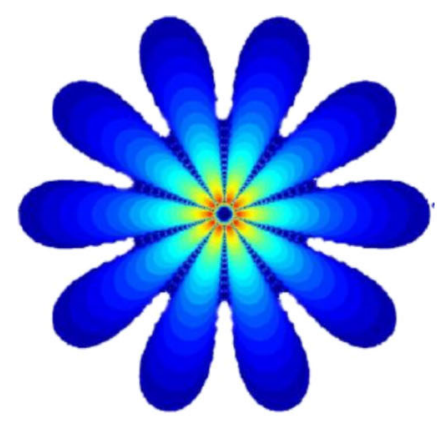

a) Basic pattern 1

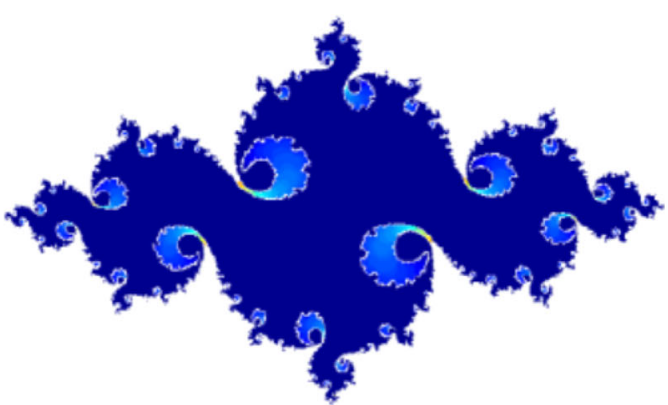

b) Basic pattern 2

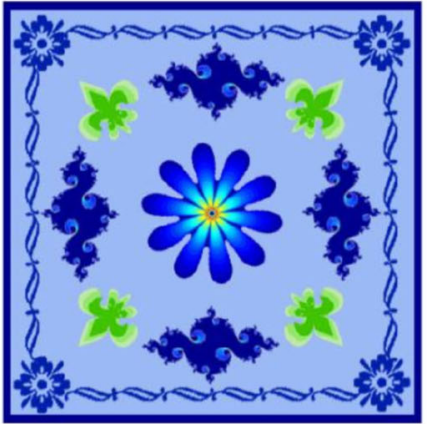

C) Silk Scarves Design

Fig. 14 Silk scarves pattern design 1 


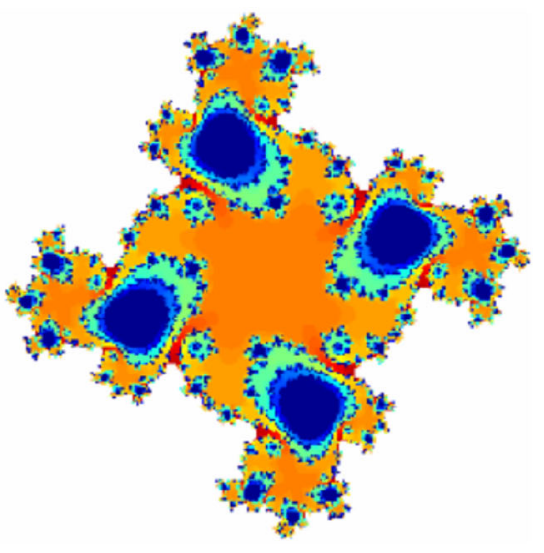

a) Basic pattern

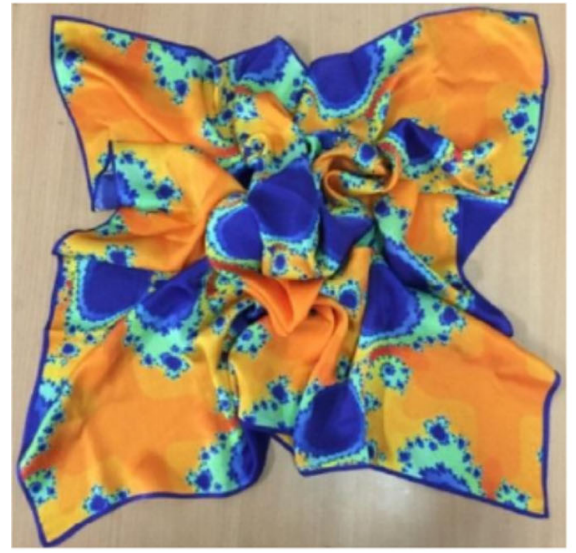

c) Physical map

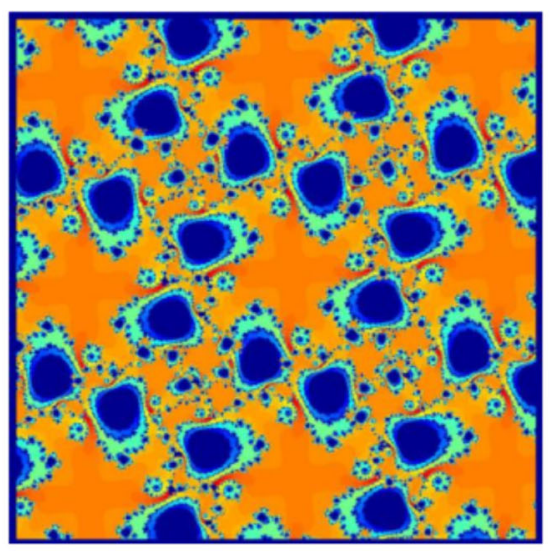

b) Silk scarves design drawings

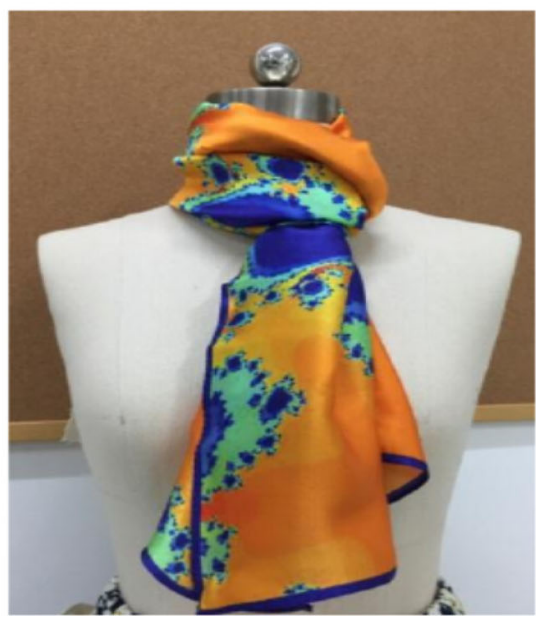

d) Wearing chart

Fig. 15 Silk scarves pattern design 2

combination, arrangement, rotation, and color change of basic patterns.

The geometric pattern figure generated by L-system is selected as the basic pattern for fabric pattern design, which is shown in Fig. 16(a), and then the pattern is designed according to the formal beauty rules of the pattern design further, to obtain the fabric pattern through continuous arrangement as shown in Fig. 16(b).

The fabric pattern shown in Fig. 17(b) is obtained by selecting the suitable basic pattern (Fig. 17a) generated by the complex dynamical system as the basic pattern for fabric pattern design, and then the secondary design on the pattern is conducted according to the formal beauty rules of pattern design. Similarly, Fig. 18(b) can be obtained based on the basic pattern as shown in Fig. 18(a).
The pattern generated by the complex dynamical system is selected as the basic pattern for fabric pattern design, which is shown in Fig. 19(a) and then the pattern is designed for the second time according to the formal beauty rule of pattern design, to obtain the fabric pattern through continuous arrangement as shown in Fig. 19(b). Finally, the fabric pattern is digitally printed and a $2-\mathrm{m}$ physical figure can be obtained as shown in Fig. 19(c) and (d).

In the design process of garment fabric printing patterns, designers should conduct design patterns according to different consumer demand and different product use, and some patterns are suitable for clothing, but not necessarily for silk scarves, thus determining the purpose of products means the general direction of pattern design is clear. In addition, color is the primary factor to attract consumers, which requires designers to have a very proper grasp of pattern color, and the reasonable 


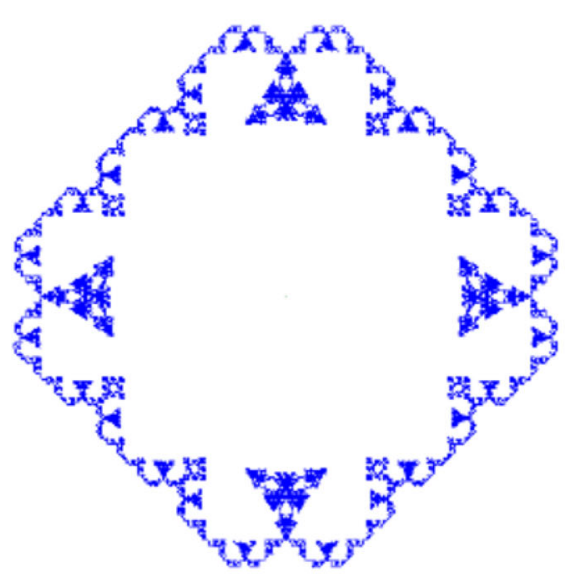

a) Basic pattern

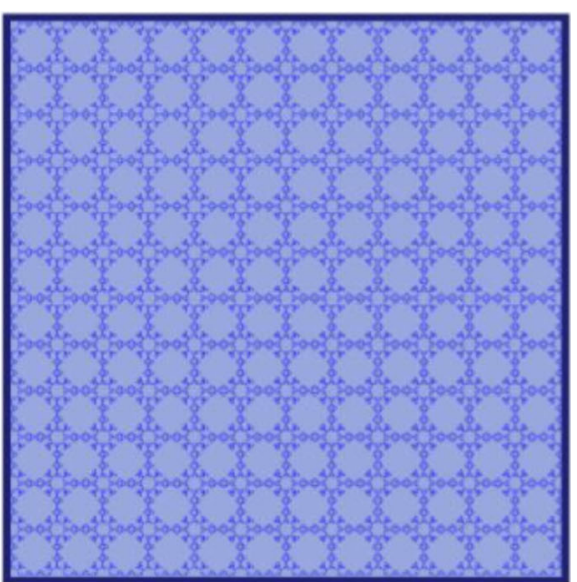

b) Fabric pattern

Fig. 16 Printing pattern design of garment fabrics 1

use can make pattern effect more colorful and form esthetic feeling more intense; otherwise, the design pattern will appear neither fish nor fowl.

\section{Conclusion}

Fractal graphics can be generated by computer programming; by changing parameters, various artistic patterns with different shapes can be generated. As a new design resource, fractal has attracted people's attention in the field of design. This paper firstly analyzes the fractal theory and L-system generation method, and then the computer pattern technology is combined with complex dynamical system theory to generate fractal art graphics by computer programming, where the generation methods of clothing pattern in complex dynamical system are analyzed in detail. Combining with computer graphics technology, a series of source codes to generate geometric patterns that can meet people's esthetic needs are compiled, to obtain the L-system design results of garment geometric patterns. Based on the aforementioned study results, a series of clothing patterns formation are presented. Finally, the fractal pattern generation theory studied in this paper is applied to conduct the actual design of silk scarves and garment fabric patterns. Based on the experimental result, it shows that it is completely feasible to design clothing fabric printing patterns based on fractal theory, and the unusual artistic patterns designed have very considerable practical value.

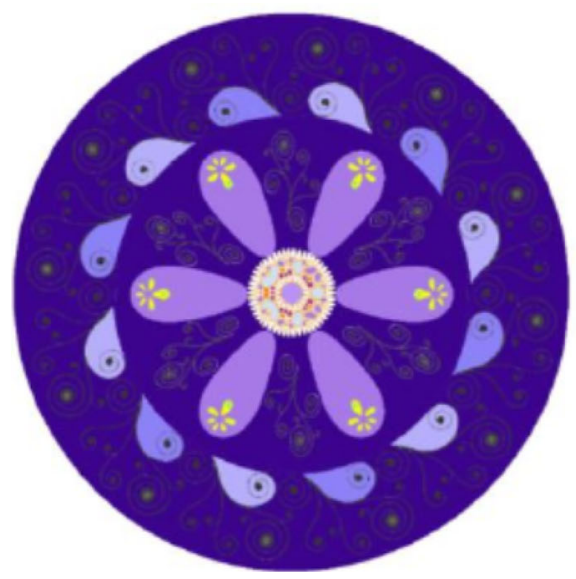

a) Basic pattern

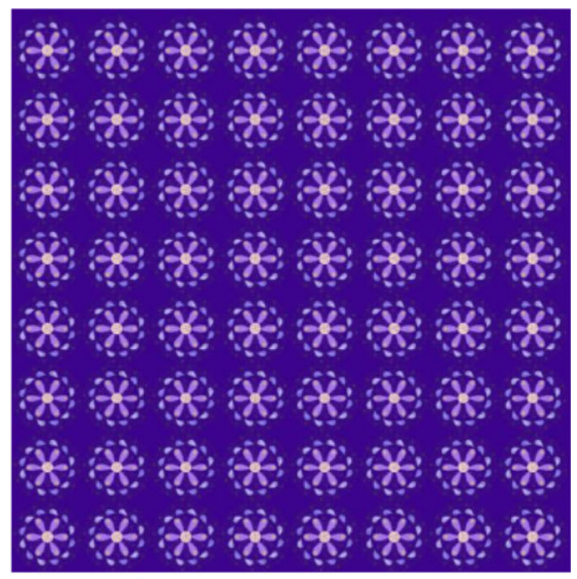

b) Fabric pattern

Fig. 17 Printing pattern design of garment fabrics 2 


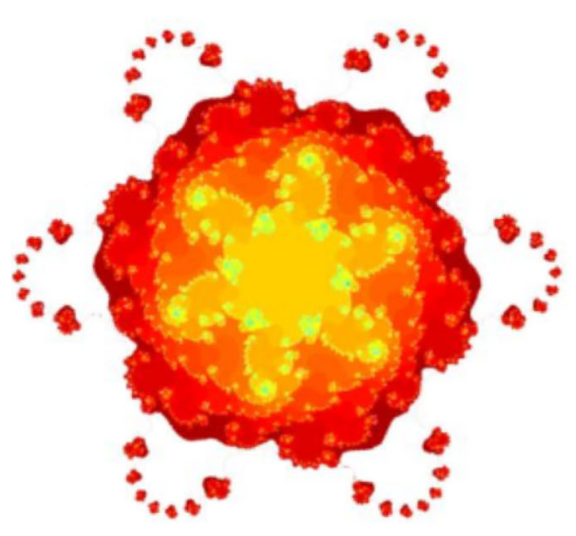

a) Basic pattern

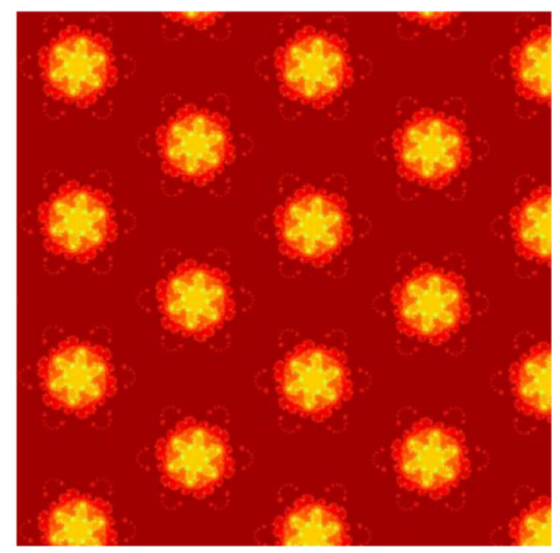

b) Fabric pattern

Fig. 18 Printing pattern design of garment fabrics 3

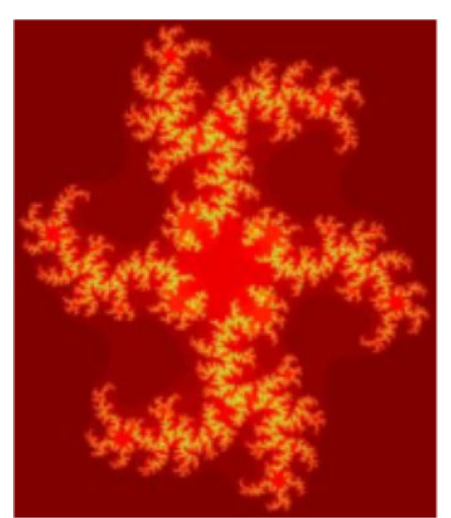

a) Basic pattern

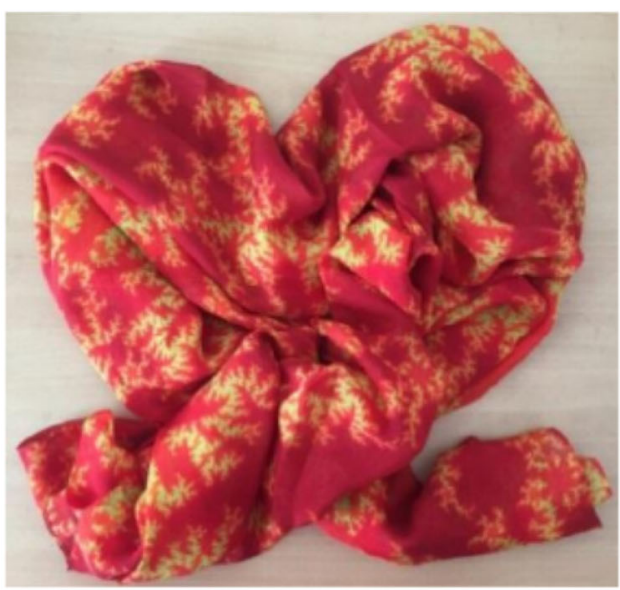

C) Physical figure 1

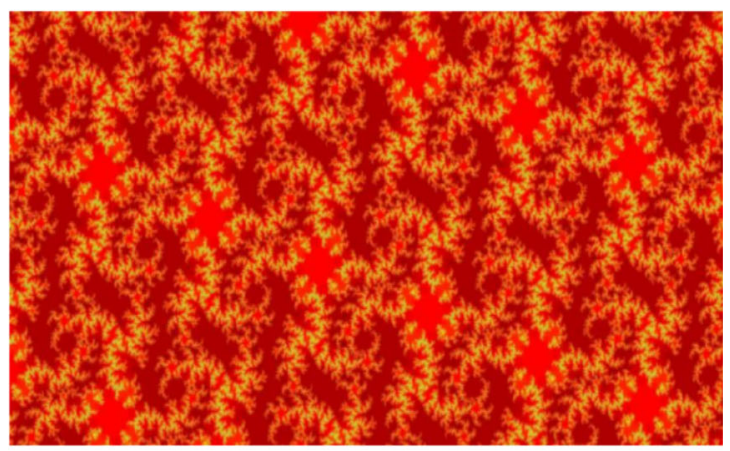

b) Fabric pattern

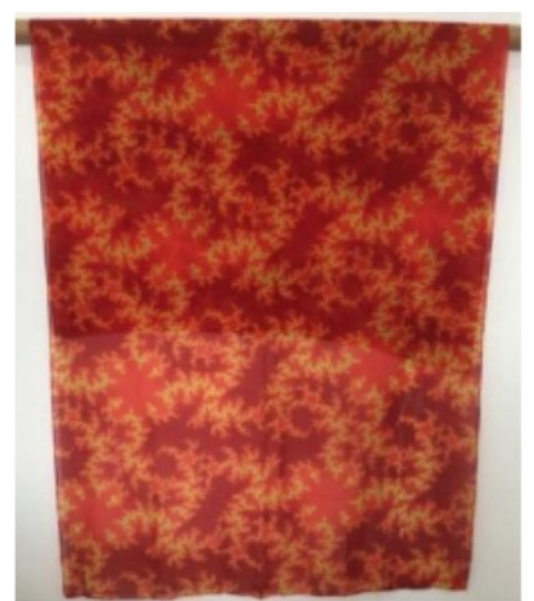

d) Physical figure 2

Fig. 19 Printing pattern design of garment fabrics 


\section{Acknowledgements}

The authors thank the editor and anonymous reviewers for their helpful comments and valuable suggestions.

\section{About the authors}

Wang Weijie received his B.S. degree in clothing design and engineering from Sichuan University, Chengdu, China, in 2016 and he is currently a M.S. candidate in clothing design and engineering in Sichuan University. His research interests include fashion $2 \mathrm{D}$ and $3 \mathrm{D} C \mathrm{CAD}$, computer graphics in fashion applications, digital human modeling and artificial intelligent applications in the fashion industry.

Zhang Gaopeng received his B.S. degree in clothing engineering from China Textile University and the M.S. degree in clothing and textile design from the Hong Kong polytechnic university in Hong Kong, China, in 2002.Since 2000, he has been an associate professor with the faculty of textile and garment engineering, Sichuan University. His research interest is mainly in the area of Fashion pattern engineering, Fashion 2D and 3D CAD and southwestern minority costume culture in China.

Yang Luming received her Ph.D. degree in Chemistry and material technology from The Tomas Bata University in Zlín, Czech, in 2011. She is currently an associate professor with National Engineering Laboratory for Clean Technology of Leather Manufacture, Sichuan University. Her research interests include shoes and health, foot biomechanics, functional leather products.

Wang Wei received the B.S. degree in Garment design and engineering from Sichuan University, China, in 2005 and the M.S. degree in Garment design and engineering from Sichuan University, China, in 2012. He is currently pursuing the Ph.D. degree in National Engineering Laboratory for Clean Technology of Leather Manufacture, Sichuan University, China. His research interest includes the computer aided garment design, three-dimensional anthropometric and fitting techniques, functional materials and functional leather products, and biomechanics of human motion.

\section{Funding}

Not applicable.

\section{Availability of data and materials}

Please contact author for data requests.

\section{Authors' contributions}

All authors took part in the discussion of the work described in this paper. All authors read and approved the final manuscript.

\section{Competing interests}

The authors declare that they have no competing interests.

\section{Publisher's Note}

Springer Nature remains neutral with regard to jurisdictional claims in published maps and institutional affiliations.

\section{Author details}

'Faculty of Textile and Garment Engineering, College of Light Industry, Textile and Food Engineering, Sichuan University, Chengdu 610065, China. ${ }^{2}$ National Engineering Laboratory for Clean Technology of Leather Manufacture, Sichuan University, Chengdu 610065, China.

Received: 25 October 2018 Accepted: 20 January 2019

Published online: 01 February 2019

\section{References}

1. R. Brouet, A. Sheffer, M.P. Cani, et al., Design preserving garment transfer. ACM Trans. Graph. 31(4), 36 (2012)

2. Z.H. Hu, Y.S. Ding, W.B. Zhang, et al., An interactive co-evolutionary CAD system for garment pattern design. Comput. Aided Des. 40(12), 1094-1104 (2008)

3. Z.G. Luo, M.M.F. Yuen, Reactive 2D/3D garment pattern design modification. Comput. Aided Des. 37(6), 623-630 (2005)

4. X.H. Yang, J.H. Sui, B. Meng, X.W. Zhang, Auto-generating uniform stochastic web images for ink-jet printing textiles. Text. Res. J. 80(18), 1942-1948 (2010)
5. B. Mandelbrot, How long is the coast of Britain? Statistical self-similarity and fractional dimension. Science 156(3775), 636-638 (1967)

6. A.P. Pentland, Fractal-based description of natural scenes. IEEE Trans. Pattern Anal. Mach. Intell. 6(6), 661-674 (1984)

7. B.B. Mandelbrot, A. Blumen, Fractal geometry: What is it, and what does it do? [and discussion]. Proc. R. Soc. Lond. 423(1864), 3-16 (1989)

8. B.B. Mandelbrot, M.J.P.T. Aizenman, Fractals: form, chance, and dimension. Phys. Today 32(5), 65-66 (1979)

9. H.X. Jiang, H.F. Wang, J.H. Liu, R.R. Pan, Development of image pattern for textile based on FFT. International Journal of Clothing Science and Technology 24(5), 295-307 (2012)

10. J. Neves, K. Janssens, M. Neves, Fractal geometry - a new tool for textile design development applications in printing. International Journal of Clothing Science and Technology 6(1), 28-36 (1994)

11. B.B. Mandelbrot, The fractal geometry of nature (W.H. Freeman, New York, 1982)

12. P. Prusinkiewicz, A. Lindenmayer, The algorithmic beauty of plants (Springerverlag, New York, 1990)

13. Prusinkiewicz P. Graphical applications of I-systems Proceedings - graphics Interface, 1986, 86: 247--253

14. J.A. Barnes, L.M.A. Koss, Julia set that is everything. Math. Mag. 76(4), 255-263 (2003)

15. C.C.L. Wang, K. Tang, Pattern computation for compression garment by a

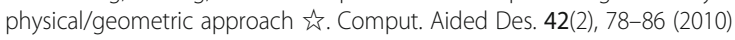

16. S. Lu, P.Y. Mok, X. Jin, A new design concept: 3D to $2 \mathrm{D}$ textile pattern design for garments. Comput. Aided Des. 89 (2017)

17. A. Bartle, D.M. Kaufman, D.M. Kaufman, et al., Physics-driven pattern adjustment for direct 3D garment editing. ACM Trans. Graph. 35(4), 50 (2016)

18. C.C.L. Wang, M.M.F. Yuen, CAD methods in garment design. Comput. Aided Des. 37(6), 583-584 (2005)

19. C.C.L. Wang, WireWarping: A fast surface flattening approach with lengthpreserved feature curves. Comput. Aided Des. 40(3), 381-395 (2008)

20. M.A. Hann, Structure and form in textile design: curriculum and bibliography. J. Text. Inst. 109(3), 285-293 (2018)

21. S. Wang, X. Yang, Generation of fractal image on complex plane and its application in textiles. Journal of Silk 54(8), 56-61 (2017) (In Chinese)

22. Y. Zhang, Q. Shuai, F. Yueying, Printing pattern designing based on general Julia set. J. Text. Inst. 28(4), 80-83 (2007) (In Chinese)

23. S. Lu, P.Y. Mok, X. Jin, From design methodology to evolutionary design: An interactive creation of marble-like textile patterns. Eng. Appl. Artif. Intel. 32(32), 124-135 (2014)

24. E. Lutton, Evolution of fractal shapes for artists and designers, Int. J. Artif. Intell. Tools. 15(04), 651-672 (2006)

25. Bergen, Steven, B. J. Ross, Automatic and interactive evolution of vector graphics images with genetic algorithms. Vis. Comput. 28(1), 35-45 (2012)

\section{Submit your manuscript to a SpringerOpen ${ }^{\circ}$ journal and benefit from:}

- Convenient online submission

- Rigorous peer review

- Open access: articles freely available online

- High visibility within the field

- Retaining the copyright to your article

Submit your next manuscript at $\boldsymbol{\nabla}$ springeropen.com 